\title{
Serum uric acid does not predict cardiovascular or all-cause mortality in type 2 diabetes: the Fremantle Diabetes Study
}

\author{
G. Ong • W. A. Davis • T. M. E. Davis
}

Received: 21 December 2009 / Accepted: 1 March 2010 /Published online: 30 March 2010

(C) Springer-Verlag 2010

\begin{abstract}
Aims/hypothesis To determine whether serum uric acid: (1) is associated with cardiovascular disease (CVD) death and/ or all-cause mortality in type 2 diabetes; and (2) consistent with published data, predicts these outcomes in older patients and those of southern European ethnicity.

Methods We studied those 1,268 (98\%) of 1,294 type 2 participants in the observational Fremantle Diabetes Study who had a fasting serum uric acid measured at baseline. Mortality data were collected over a mean $( \pm \mathrm{SD}) 10.3 \pm$ 3.9 years. Cox proportional hazards modelling was used to determine independent baseline predictors of CVD and allcause death including fasting serum uric acid as a continuous variable and quartiles.

Results During follow up, 525 deaths occurred (41.4\% of the cohort) of which $271(51.6 \%)$ were attributed to CVD. In univariate analyses, patients in the highest uric acid quartile had the greatest CVD and all-cause mortality $(p=0.007$ and $p=0.001$ ). After adjustment for significant variables in the most parsimonious model, baseline serum uric acid was not an independent associate of CVD or all-cause mortality whether entered as a continuous variable (HR 1.11 [95\% CI 0.96-1.27] and 1.10 [95\% CI $0.98-1.22]$ for a $0.1 \mathrm{mmol} / \mathrm{l}$ increase, respectively) or as quartiles $(p>0.10)$. Analyses of 638 patients $>65$ years of age and 231 of southern European ethnicity produced similar results.

Conclusions/interpretation Serum uric acid was not an independent predictor of CVD or all-cause mortality in our community-based type 2 patients. Fasting serum uric
\end{abstract}

G. Ong $•$ W. A. Davis $\cdot$ T. M. E. Davis $(\bowtie)$

School of Medicine and Pharmacology, Fremantle Hospital,

University of Western Australia,

P.O. Box 480, Fremantle, WA 6959, Australia

e-mail: tdavis@cyllene.uwa.edu.au acid concentrations do not appear to be prognostically useful in type 2 diabetes.

Keywords Cardiovascular disease $\cdot$ Mortality Type 2 diabetes $\cdot$ Uric acid
Abbreviations
ACR Urinary albumin:creatinine ratio
CVD Cardiovascular disease
eGFR Estimated GFR
FDS Fremantle Diabetes Study
IQR Interquartile range
PAD Peripheral arterial disease

\section{Introduction}

Although there are reported positive associations between serum uric acid concentration and both cardiovascular disease (CVD) and its risk factors [1], it is unclear whether this reflects causation or confounding. In the case of type 2 diabetes, a recent Italian cohort study found that serum uric acid was independently associated with CVD mortality [2], but those with a prior history of CVD, malignancy, severe chronic airway disease and/or end-stage renal disease were excluded, and there was no significant relationship with allcause death. In two smaller studies in diabetic patients, serum uric acid was independently associated with all-cause mortality in a sample at relatively high CVD risk [3] and with CVD mortality in the elderly in a 'J-shaped' relationship [4]. Studies in other disease states have found the same J-shaped relationship with CVD outcomes [5-7], suggesting that the pathophysiological effects of uricaemia are concentration-dependent. 
There is a need for further longitudinal population-based studies that assess the prognostic significance of serum uric acid concentrations, including the possibility of non-linear associations. We have, therefore, investigated the relationship between serum uric acid and CVD and all-cause mortality in a large well-characterised representative community-based cohort of type 2 patients.

\section{Methods}

Patients The Fremantle Diabetes Study (FDS) was a longitudinal observational cohort study of patients from a postcode-defined urban community of 120,097 people in the state of Western Australia (WA). Descriptions of recruitment, sample characteristics, including classification of diabetes type and details of non-recruited patients, have been published elsewhere [8]. Of 2,258 diabetic patients identified between 1993 and 1996, 1,426 (63\%) were recruited to the FDS and 1,294 had type 2 diabetes. Eligible patients who declined participation were a mean of 1.4 years older than participants, but their sex distribution, the proportion with type 2 diabetes and their use of blood glucose-lowering therapies were similar [8]. The FDS protocol was approved by the Human Rights Committee at Fremantle Hospital and all individuals gave informed consent before participation.

Baseline and annual assessments The assessment of each patient at study entry and at each annual review included a comprehensive standardised questionnaire and physical examination [8]. We collected data relating to: (1) demographic characteristics including self-described ethnicity (assessed principally from self-description, with other contributory variables including country of birth/parents' birth and language spoken at home); (2) diabetes-specific data including vascular and other complications; (3) current lifestyle measures; (4) access to healthcare; (5) knowledge of diabetes; (6) other past illnesses and their treatment (including all drugs with known effects on serum uric acid such as aspirin, diuretics and allopurinol); (7) general and diabetes-specific quality of life; (8) cognitive function and activities of daily living; and (9) costs of diabetes. Physical examination comprised anthropometric measures, cardiovascular assessment including detailed foot assessment and a 12-lead electrocardiogram, respiratory assessment, neurological assessment with special reference to the feet, and ophthalmological assessment.

Biochemical tests were performed using standard automated methods in a single laboratory. Fasting venous blood samples were centrifuged promptly and separated serum and plasma were stored at $-80^{\circ} \mathrm{C}$. Fasting serum uric acid was measured, along with other routine serum biochemistry including serum glucose, cholesterol, HDL-cholesterol and triacylglycerol, using enzymatic methods with reagents supplied by Roche Diagnostics on a Hitachi 911 analyser (Roche Diagnostics, Castle Hill, NSW, Australia). The inter-assay $\mathrm{CV}$ values for serum uric acid were $0.9 \%$ at $0.60 \mathrm{mmol} / 1$ and $1.9 \%$ at $0.18 \mathrm{mmol} / \mathrm{l}$. $\mathrm{HbA}_{1 \mathrm{c}}$ was measured by cation-exchange HPLC using a Mono S HR 5/5 column (Amersham Biosciences, Castle Hill, NSW, Australia). Urine albumin levels were measured using a Hitachi 911 analyser by an immunoturbidimetric assay (Roche Diagnostics).

Baseline complications were identified using standard definitions [9] based on data obtained from: (1) selfreport; (2) physical examination and laboratory testing; and (3) the WA Data Linkage System [10] which records all hospitalisations in the state. Microalbuminuria was defined as a urinary albumin:creatinine ratio (ACR) of $\geq 3.0 \mathrm{mg} / \mathrm{mmol}$. The estimated GFR (eGFR) was calculated from the serum creatinine [11]. Neuropathy was defined using the clinical portion of the Michigan Neuropathy Screening Instrument. Retinopathy was defined as one microaneurysm in either eye or worse and/or evidence of previous laser treatment on direct and/or indirect ophthalmoscopy through dilated pupils, and/or more detailed ophthalmological data in patients assessed by a specialist ophthalmologist.

Patients were classified as having CHD at baseline if there was a history of: (1) myocardial infarction (selfreported event or documented hospitalisation between the beginning of January 1993 and study entry, a period between 0.3 and 3.5 years); (2) angina (self-reported symptoms, prescription of anti-anginal medications and/or hospitalisation with documented angina between January 1993 and study entry); or (3) coronary artery bypass grafting or angioplasty (self-reported event or documented hospitalisation between January 1993 and study entry). Cerebrovascular disease was defined as a history of stroke and/or transient ischaemic attack as a self-reported event or documented hospitalisation between January 1993 and study entry. Peripheral arterial disease (PAD) was defined as an ankle brachial index $\leq 0.90$ or the presence of a diabetes-related lower-extremity amputation.

Mortality ascertainment All deaths in WA are also recorded in the WA Data Linkage System [10] which was used to provide FDS patient outcomes from the beginning of the study until end of June 2007. Causes of death were reviewed independently by two physicians and classified under the system used in the UK Prospective Diabetes Study [12]. In the case of discrepant coding, case notes were consulted and a consensus obtained. CVD death was taken as that attributed to $\mathrm{CHD}$, cardiac failure, cerebrovascular or PAD or sudden death. 
Statistical analysis The computer package SPSS for Windows (version 15.0) was used for statistical analysis. Rightskewed variables were transformed using the natural logarithm $(\log )$. Data are presented as proportions, means \pm $\mathrm{SD}$, geometric mean (SD range), or, in the case of variables which did not conform to a normal or log-normal distribution, median with inter-quartile range (IQR). For independent samples, two-way comparisons for proportions were by Fisher's exact test, for normally distributed variables by Student's $t$ test, and for non-normally distributed variables by the Mann-Whitney $U$ test. Multiple comparisons for proportions were by Fisher's exact test or $\chi^{2}$ test, for normally distributed variables by one-way ANOVA, and for non-normally distributed variables by the Kruskal-Wallis $H$ test. A two-tailed significance level of $p<0.05$ was used throughout.

Kaplan-Meier analysis was used to assess all-cause mortality and CVD death by baseline serum uric acid quartile with comparisons by logrank test. Cox proportional hazards modelling (forward conditional variable entry and removal with $p<0.05$ and $>0.10$, respectively) was used to determine independent baseline predictors of all-cause and CVD mortality. All clinically plausible variables were considered for entry into the models, including demographic and diabetes-related factors, the presence of other diabetic complications including renal impairment (eGFR $<60 \mathrm{ml} \mathrm{min}^{-1}$ $1.73 \mathrm{~m}^{-2}$ ), cardiovascular risk factors, and serum uric acid as both a continuous variable and as quartiles. The validity of the proportional hazards assumption was assessed from log $(-\log [$ survival] $)$ curves and examination of time-dependent covariates.

\section{Results}

Baseline patient characteristics Of the 1,294 type 2 FDS participants, 1,268 (98.0\%) had serum uric acid measured at study entry. These 1,268 patients were of mean \pm SD age $64.1 \pm 11.3$ years, $48.6 \%$ were men and their diabetes had been diagnosed a median 4.0 (IQR 1.0-9.0) years previously. The mean serum uric acid was $0.35 \pm 0.10 \mathrm{mmol} / \mathrm{l}$.

Baseline patient characteristics are summarised by quartile of fasting serum uric acid in Table 1. There were trends in variables associated with insulin resistance, with patients in the higher quartiles more likely to be older, male, to have a high BMI, waist circumference, fasting serum triacylglycerol and urinary ACR, to have a low fasting serum HDL-cholesterol, and to have had a history of gout. CHD, renal impairment and ACE inhibitor use were more prevalent in the highest quartile group. In the lower serum uric acid quartile groups, there were larger proportions of patients with a non-Anglo-Celt ethnic background, a longer median diabetes duration and higher mean fasting serum glucose and $\mathrm{HbA}_{1 \mathrm{c}}$ levels. The proportions of current smokers were similar across the quartile groups.

Cardiovascular and all-cause mortality There were 525 deaths $(41.4 \%)$ during a total of 13,113 patient-years $(10.3 \pm 3.9$ years $)$ of follow-up, of which approximately half $(271$ or $51.6 \%)$ were attributed to CVD. In univariate analysis, there was a significant difference between quartiles of serum uric acid and their association with CVD mortality, with the lowest survival in patients with a serum uric acid concentration in the highest quartile (logrank test $p=0.007$; Fig. 1). With serum uric acid as a continuous variable, the univariate HR for an increase of $0.1 \mathrm{mmol} / 1$ was $1.28(95 \%$ CI $1.13-1.44)(p<0.001)$. Using the first quartile as reference, the HR for the highest quartile was 1.79 $(95 \%$ CI $1.28-2.50) \quad(p=0.001)$; the second and third quartiles were not significantly different from the reference quartile or each other, or from the highest quartile $(p>0.05)$. A similar pattern was also found for all-cause mortality $(p=0.001)$. With serum uric acid as a continuous variable, the univariate $\mathrm{HR}$ for an increase of $0.1 \mathrm{mmol} / \mathrm{l}$ was 1.23 (95\% CI 1.13-1.34) $(p<0.001)$. Using the first quartile as reference, the HR for the highest quartile was 1.59 (95\% CI $1.26-2.01)(p<0.001)$; the second and third quartiles were not significantly different from the reference quartile or each other, or from the highest quartile $(p>0.05)$.

The proportional hazards assumption, assessed from log $(-\log [$ survival $])$ curves and examination of time-dependent covariates, was valid for all variables in all modelling performed. After adjustment for significant variables, including those relating to demographics, cardiovascular risk factors and cardiovascular/other medications, there was no significant association between serum uric acid and CVD death (Table 2). In addition, a receiver operating characteristic (ROC) curve showed that serum uric acid was a poor predictor of CVD mortality (AUC 0.553 ). There was similarly no independent association between serum uric acid and all-cause mortality (Table 3 ) and the ROC AUC was 0.552 .

In Cox models restricted to (1) men, (2) women, (3) patients aged $>65$ years, (4) those of southern European ethnic background, (5) all but the 125 participants $(10 \%)$ who were not of wholly Europid descent, (6) those without a history of CVD at baseline, and (7) those with a history of CVD at baseline, there was no independent association between serum uric acid, included as a continuous variable or as quartiles, and either all-cause or CVD mortality ( $p>$ 0.08 in each case). In addition, excluding retinopathy, neuropathy, $\log (\mathrm{ACR})$ and eGFR $<60 \mathrm{ml} \mathrm{min}^{-1} 1.73 \mathrm{~m}^{-2}$ as potential predictors of outcome did not result in uric acid entering the models as a significant independent predictor (data not shown). 
Table 1 Baseline characteristics of 1,268 FDS participants with type 2 diabetes by serum uric acid quartile

\begin{tabular}{|c|c|c|c|c|c|}
\hline Characteristic & $\begin{array}{l}\text { Quartile } 1 \\
(\leq 0.28 \mathrm{mmol} / \mathrm{l})\end{array}$ & $\begin{array}{l}\text { Quartile } 2 \\
(>0.28-0.34 \mathrm{mmol} / \mathrm{l})\end{array}$ & $\begin{array}{l}\text { Quartile } 3 \\
(>0.34-0.40 \mathrm{mmol} / \mathrm{l})\end{array}$ & $\begin{array}{l}\text { Quartile } 4 \\
(>0.40 \mathrm{mmol} / \mathrm{l})\end{array}$ & $p$ value \\
\hline Number & 368 & 323 & 279 & 298 & \\
\hline Age (years) & $62.9 \pm 11.7$ & $63.8 \pm 11.3$ & $64.8 \pm 10.8^{*}$ & $65.2 \pm 11.1 * *$ & 0.038 \\
\hline Sex $(\%$ male $)$ & 34.8 & $52.0 * * *$ & $53.4 * * *$ & $57.4 * * *$ & $<0.001$ \\
\hline \multicolumn{6}{|l|}{ Ethnicity (\%) } \\
\hline Anglo-Celt/southern European/other European & $58.2 / 24.5 / 7.3$ & $65.0 / 18.3 / 8.4$ & $67.0 * / 14.7 / 10.4$ & $64.4 * / 13.8 / 8.4$ & \\
\hline Asian & 3.8 & 3.7 & 1.8 & 3.4 & \\
\hline Aboriginal & 1.4 & 0.9 & 1.1 & 2.3 & \\
\hline Other & 4.9 & 3.7 & 5.0 & 7.7 & \\
\hline Education beyond primary level (\%) & 72.1 & 73.1 & 77.2 & 74.7 & 0.51 \\
\hline Not fluent in English (\%) & 19.0 & 15.2 & 14.0 & 11.8 & 0.07 \\
\hline Currently married/de facto relationship (\%) & 63.0 & 70.6 & 65.9 & 63.5 & 0.16 \\
\hline Smoking status, never/ex-/current (\%) & $54.5 / 30.7 / 14.8$ & $42.5 * * / 40.6 / 17.0$ & $42.1 * * / 42.4 / 15.5$ & $38.3 * * * / 49.5 / 12.2$ & $<0.001$ \\
\hline Any exercise in past 2 weeks (\%) & 74.0 & 74.6 & 72.6 & 66.4 & 0.10 \\
\hline Alcohol consumption (standard drinks/day) & $0[0-0.3]$ & $0[0-0.8]^{* *}$ & $0[0-0.8]^{*}$ & $0[0-0.8]^{* *}$ & 0.015 \\
\hline Diabetes duration (years) & $5.0[1.8-10.0]$ & $4.0[1.0-9.0]$ & $4.0[1.0-9.0]^{*}$ & $2.5[0.5-6.0]^{* * *}$ & $<0.001$ \\
\hline Fasting serum glucose (mmol/l) & $9.6[7.2-12.5]$ & $8.8[7.1-11.1]^{* *}$ & $8.0[6.8-10.2]^{* * *}$ & $7.6[6.5-9.4]^{* * *}$ & $<0.001$ \\
\hline $\mathrm{HbA}_{1 \mathrm{c}}(\%)$ & $8.2[6.7-9.7]$ & $7.6[6.6-8.9]^{* *}$ & $7.2[6.3-8.2]^{* * *}$ & $7.0[6.1-8.3]^{* * *}$ & $<0.001$ \\
\hline $\begin{array}{l}\text { Diabetes treatment, diet/oral agents/insulin } \pm \text { oral } \\
\text { agents }(\%)\end{array}$ & $27.8 / 56.7 / 15.5$ & $31.8 / 57.0 / 11.2$ & $33.7 / 55.9 / 10.4$ & $35.1 / 55.4 / 9.5$ & 0.15 \\
\hline BMI $\left(\mathrm{kg} / \mathrm{m}^{2}\right)$ & $28.7 \pm 5.5$ & $29.2 \pm 5.2$ & $30.3 \pm 5.4 * * *$ & $30.3 \pm 5.5 * * *$ & $<0.001$ \\
\hline Abdominal obesity, by waist circumference ${ }^{\mathrm{a}}(\%)$ & 56.8 & 61.9 & $71.3 * * *$ & $69.5^{* *}$ & $<0.001$ \\
\hline Systolic BP (mmHg) & $149 \pm 24$ & $152 \pm 23 * *$ & $152 \pm 24 * *$ & $151 \pm 23$ & 0.46 \\
\hline Diastolic BP (mmHg) & $79 \pm 11$ & $82 \pm 11$ & $82 \pm 11$ & $79 \pm 11$ & 0.001 \\
\hline Taking antihypertensive medication (\%) & 41.0 & 46.4 & $52.7 * *$ & $67.8 * * *$ & $<0.001$ \\
\hline Taking an ACE inhibitor (\%) & 14.9 & 18.4 & $22.6^{*}$ & $33.6^{* * *}$ & $<0.001$ \\
\hline Total serum cholesterol (mmol/l) & $5.5 \pm 1.1$ & $5.5 \pm 1.1$ & $5.5 \pm 1.1$ & $5.4 \pm 1.2$ & 0.85 \\
\hline Serum HDL-cholesterol (mmol/l) & $1.14 \pm 0.33$ & $1.07 \pm 0.34 * *$ & $1.02 \pm 0.28 * * *$ & $1.00 \pm 0.31 * * *$ & $<0.001$ \\
\hline Serum triacylglycerol (mmol/l) & $1.7(0.9-3.0)$ & $1.9(1.1-3.3)^{* *}$ & $2.0(1.2-3.4)^{* * *}$ & $2.1(1.2-3.7)^{* * *}$ & $<0.001$ \\
\hline Taking lipid-lowering medication (\%) & 8.7 & 7.1 & 12.6 & $14.5^{*}$ & 0.010 \\
\hline Regular aspirin use, $\geq 75$ mg/day (\%) & 16.1 & 21.1 & $22.6^{*}$ & $28.5^{* * *}$ & 0.002 \\
\hline Self-reported gout, past history/current (\%) & $1.9 / 7.1$ & $4.0 / 7.1$ & $7.9 / 12.2 * * *$ & $15.1 / 14.4^{* * *}$ & $<0.001$ \\
\hline Taking xanthine oxidase inhibitor (\%) & 7.3 & 5.9 & 7.5 & 5.4 & 0.63 \\
\hline $\mathrm{ACR}(\mathrm{mg} / \mathrm{mmol})$ & $2.8(0.8-10.0)$ & $2.7(0.7-10.4)$ & $3.1(0.7-13.5)$ & $4.3(0.8-25.1)^{* * *}$ & $<0.001$ \\
\hline eGFR $<60 \mathrm{ml} \mathrm{min}^{-1} 1.73 \mathrm{~m}^{-2}(\%)$ & 8.5 & 13.9 & 24.7 & 41.9 & $<0.001$ \\
\hline Peripheral neuropathy $(\%)$ & 26.8 & 29.8 & 32.7 & 36.5 & 0.06 \\
\hline Any retinopathy $(\%)$ & 18.7 & 15.8 & 14.1 & 17.1 & 0.48 \\
\hline CHD $(\%)$ & 22.0 & 25.4 & 27.6 & $37.2 * * *$ & $<0.001$ \\
\hline Cerebrovascular disease $(\%)$ & 7.3 & 11.8 & 9.0 & $13.4^{*}$ & 0.046 \\
\hline $\operatorname{PAD}(\%)$ & 27.0 & 27.9 & 28.3 & 34.5 & 0.16 \\
\hline
\end{tabular}

Values are percentages, means $\pm \mathrm{SD}$, geometric means (SD range), or, in the case of variables which did not conform to a normal or log-normal distribution, median [IQR]

${ }^{\mathrm{a}}$ Men $\geq 94 \mathrm{~cm}$; women $\geq 80 \mathrm{~cm}$

${ }^{*} p<0.05,{ }^{* *} p<0.01,{ }^{* * *} p<0.001$ compared with quartile 1 (unadjusted for multiple comparisons)

\section{Discussion}

The present study shows that baseline serum uric acid is not independently associated with either CVD mortality or all- cause death after adjustment for other risk factors in a community-based cohort of patients with type 2 diabetes followed for a mean of $>10$ years. This situation pertained regardless of whether serum uric acid was included in Cox 


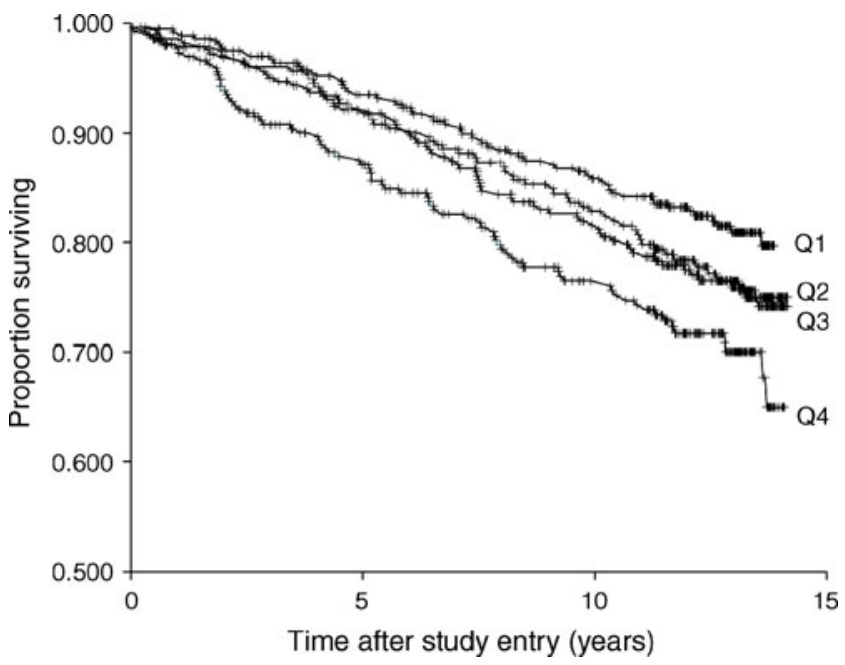

Fig. 1 Kaplan-Meier plots of survival from cardiovascular disease stratified by serum uric acid quartiles (Q1-Q4) at baseline

proportional hazard models as a continuous variable or as quartiles. Because of previous reports that uricaemia is of prognostic significance in older patients [4] and in an Italian population-based sample [2], we performed separate analyses in the patients in our cohort who were $>65$ years and from a southern European background. In neither case was serum uric acid an independent determinant of outcome. Our data suggest that serum uric acid concentrations have a limited influence on CVD complicating type 2 diabetes.

In the Verona Diabetes Study [2] which involved 2,726 type 2 patients followed for a mean of 4.7 years, each SD increment in serum uric acid $(0.095 \mathrm{mmol} / \mathrm{l})$ was associated with an independent $27 \%$ increase in the risk of CVD death in a fully adjusted Cox model. However, these authors excluded patients with a prior history of CVD, and nonrenal microvascular complications of diabetes were not included in any of the analyses. In the present study, retinopathy, neuropathy, coronary disease and PAD were independent determinants of CVD death and/or all-cause mortality, while we also had near-complete baseline and outcome data for our patients. Since the $28 \%$ increased risk of CVD death per $0.1 \mathrm{mmol} / \mathrm{l}$ rise in serum uric acid in our univariate analyses declined to a non-significant $11 \%$ excess in the fully adjusted model (with the upper 95\% CI equivalent to the Verona Study $27 \%$ point estimate [2]), it is likely that the difference in the range of data available for analyses helps explain the discrepant findings in the two studies. In addition, the Verona Diabetes Study patients were older and had been diabetic for a longer duration than those in the FDS despite exclusion of those with prior CVD, and may have been less representative of type 2 diabetes in the community as a result.

Elevated serum uric acid concentrations are found in individuals with the metabolic syndrome [1] and the patients in our highest quartile were most likely to have the features of this condition. Our data suggest that the clustering of established CVD risk factors such as dyslipidaemia in these patients is the prime determinant of outcome and that additional variables including serum uric acid add little to predictive models [13]. There were greater numbers of patients from southern and other parts of
Table 2 Independent determinants of time to cardiovascular mortality
The most parsimonious model is shown together with HRs and 95\% CIs, and, after adjustment for the other variables, for serum uric acid as a continuous variable and as quartiles

${ }^{\text {a }}$ A 2.72 -fold increase in ACR corresponds to an increase of 1.0 in $\log (\mathrm{ACR})$

\begin{tabular}{llr}
\hline Variable & HR $(95 \%$ CI $)$ & $p$ value \\
\hline Age (increase of 10 years) & $2.78(2.31-3.36)$ & $<0.001$ \\
Male sex & $1.84(1.40-2.42)$ & $<0.001$ \\
Aboriginal ethnicity & $3.58(1.42-9.05)$ & 0.002 \\
HbA $_{1 \mathrm{c}}$ (increase of 1.0\%) & $1.09(1.004-1.18)$ & 0.040 \\
Supine systolic BP (increase of $10 \mathrm{mmHg})$ & $0.92(0.87-0.98)$ & 0.015 \\
ACE inhibitor use & $1.59(1.18-2.14)$ & 0.002 \\
Log(ACR) & $1.20(1.10-1.32)$ & $<0.001$ \\
Retinopathy & $1.83(1.33-2.53)$ & $<0.001$ \\
Peripheral neuropathy & $1.89(1.44-2.50)$ & $<0.001$ \\
CHD & $1.94(1.48-2.55)$ & $<0.001$ \\
Cerebrovascular disease & $1.54(1.08-2.20)$ & 0.018 \\
PAD & $1.79(1.36-2.35)$ & $<0.001$ \\
After adjustment for the above variables & & 0.43 \\
Serum uric acid (increase of $0.1 \mathrm{mmol} / \mathrm{l})$ & $1.06(0.92-1.22)$ & \\
Serum uric acid (quartiles) & & 0.39 \\
1 (reference) & 1 & \\
2 & $1.18(0.81-1.73)$ & 0.61 \\
3 & $0.90(0.59-1.36)$ & 0.39 \\
4 & $1.19(0.80-1.76)$ & \\
\hline
\end{tabular}


Table 3 Independent determinants of time to all-cause mortality

The most parsimonious model is shown together with HRs and 95\% CIs, and, after adjustment for the other variables, for serum uric acid as a continuous variable and as quartiles

${ }^{\text {a }}$ A 2.72 -fold increase in serum triacylglycerol or ACR

corresponds to an increase of 1.0 in $\log (\mathrm{ACR})$ or $\log ($ serum triacylglycerol)

\begin{tabular}{llr}
\hline Variable & HR $(95 \% \mathrm{CI})$ & $p$ value \\
\hline Age (increase of 10 years) & $2.01(1.77-2.29)$ & $<0.001$ \\
Male sex & $1.77(1.45-2.16)$ & $<0.001$ \\
Asian ethnicity & $0.40(0.18-0.90)$ & 0.027 \\
Aboriginal & $2.44(1.18-5.04)$ & 0.016 \\
BMI (increase of $\left.1 \mathrm{~kg} / \mathrm{m}^{2}\right)$ & $0.97(0.95-0.99)$ & 0.007 \\
Any exercise in past 2 weeks & $0.69(0.56-0.85)$ & $<0.001$ \\
Log(serum triacylglycerol) & $\mathrm{a}$ & 0.022 \\
Lipid-lowering medication use & $0.80(0.67-0.97)$ & 0.007 \\
ACE inhibitor use & $0.60(0.42-0.87)$ & $<0.001$ \\
Log(ACR) & $1.49(1.20-1.84)$ & $<0.001$ \\
Any retinopathy & $1.20(1.12-1.27)$ & 0.001 \\
Peripheral neuropathy & $1.51(1.20-1.91)$ & $<0.001$ \\
CHD & $1.70(1.39-2.08)$ & $<0.001$ \\
PAD & $1.77(1.45-2.17)$ & $<0.001$ \\
After adjustment for the above variables & $1.59(1.31-1.94)$ & 0.32 \\
Serum uric acid (increase of $0.1 \mathrm{mmol} / \mathrm{l})$ & & 0.28 \\
Serum uric acid (quartiles) & $1.05(0.95-1.17)$ & \\
1 (reference) & & 0.92 \\
2 & 1 & $1.01(0.78-1.33)$ \\
3 & $0.89(0.67-1.19)$ & \\
4 & $1.16(0.88-1.54)$ &
\end{tabular}

Europe amongst those in the lower quartiles in association with a longer diabetes duration and worse glycaemic control. We have shown previously that the southern Europeans in our cohort had a longer diabetes duration and higher $\mathrm{HbA}_{1 \mathrm{c}}$ than the Anglo-Celts [14]. A survivor effect might also explain why longer diabetes duration was associated with less evidence of metabolic syndrome and lower rates of CHD in the lower serum uric acid quartiles, while previously published FDS data have shown that older patients have lower $\mathrm{HbA}_{1 \mathrm{c}}$ levels [15].

Therapies for CVD risk factors might confound an association between serum uric acid and mortality. In the Framingham Heart Study general population sample, there was no independent association between baseline serum uric acid and cardiac or all-cause mortality after adjustment for diuretic use [16]. Nevertheless, other studies have shown a positive association with cardiac mortality when diuretic use was allowed for [3, 17]. Since non-diuretic therapies for CVD such as statins and ACE inhibitors can raise or lower serum uric acid concentrations [18], we included the main CVD treatments in our analyses but they did not alter the non-significant relationship between serum uric acid and CVD death. As in the Verona Diabetes Study [2], we did not find that use of xanthine oxidase inhibitors was associated with CVD death. This suggests that lowering serum uric acid concentrations in this way does not have beneficial CVD effects. Xanthine oxidase produces reactive oxygen species which promote endothelial damage and reduce nitrous oxide-related vasodilatation [19], effects that appear independent of serum uric acid concentrations [1]. Trials assessing the CVD benefits of drugs such as allopurinol are awaited.

In the CArdiovascular STudy in the ELderly (CASTEL) study [4], serum uric acid was independently associated with CVD death in type 2 patients $>65$ years of age in a J-shaped association, with the second tertile having lowest adjusted relative risk. We could find no evidence for such a relationship in our total sample or in patients $>65$ years of age despite the fact that we had greater numbers of older patients than the CASTEL study (638 vs 581, respectively). Similarly, we did not find the independent association between serum uric acid and all-cause mortality reported by Ioachimescu et al. [3] in a retrospective study of 535 type 2 patients. The patients in this latter study were selected (self- or physician-referred to a CVD risk assessment clinic) and limited follow-up may have biased ascertainment of events.

Our study also had limitations. As in most such studies, we did not consider changes in serum uric acid, or in CVD therapies and those influencing uricaemia, during followup. However, the strengths of our study include a large sample size, its representative nature and a relatively long duration of follow-up. In addition, the WA Data Linkage System captures both public and private hospital admissions in Western Australia [10], with low rates of coding errors [20] and migration out of the state [21]. 
The pathophysiological complexity of the relationship between serum uric acid and CVD is illustrated by its role as a putative antioxidant agent [19] with neuroprotective properties [22], observations set against the possibility that it may act as a pro-oxidant in certain circumstances [23] and a variety of in vitro and animal studies highlighting its potential adverse CVD effects [1]. Differences in variables such as age [24], renal function [1] and CVD therapies [18], which alter both serum uric acid concentrations and independently mediate CVD risk, may explain why the results of epidemiological studies of CVD in the general population are, as in the case of type 2 diabetes, conflicting $[16,25-27]$. Further studies are needed to clarify the role of uricaemia in diabetic macroangiopathy, but our data do not suggest that measurement of fasting serum uric acid concentrations provides useful prognostic information in type 2 diabetes.

Acknowledgements The FDS was funded by the Raine Foundation, University of Western Australia. T. M. E. Davis is supported by a National Health and Medical Research Council of Australia Practitioner Fellowship.

Duality of interest The authors declare that there is no duality of interest associated with this manuscript.

\section{References}

1. Feig DI, Kang DH, Johnson RJ (2008) Uric acid and cardiovascular risk. N Engl J Med 359:1811-1821

2. Zoppini G, Targher G, Negri C et al (2009) Elevated serum uric acid concentrations independently predict cardiovascular mortality in type 2 diabetic patients. Diabetes Care 32:1716-1720

3. Ioachimescu AG, Brennan DM, Hoar BM, Kashyap SR, Hoogwerf BJ (2007) Serum uric acid, mortality and glucose control in patients with Type 2 diabetes mellitus: a PreCIS database study. Diabet Med 24:1369-1374

4. Mazza A, Zamboni S, Rizzato E et al (2007) Serum uric acid shows a J-shaped trend with coronary mortality in non-insulindependent diabetic elderly people. The CArdiovascular STudy in the ELderly (CASTEL). Acta Diabetol 44:99-105

5. Hsu SP, Pai MF, Peng YS, Chiang CK, Ho TI, Hung KY (2004) Serum uric acid levels show a 'J-shaped' association with allcause mortality in haemodialysis patients. Nephrol Dial Transplant 19:457-462

6. Suliman ME, Johnson RJ, Garcia-Lopez E et al (2006) J-shaped mortality relationship for uric acid in CKD. Am J Kidney Dis 48:761-771

7. Verdecchia P, Schillaci G, Reboldi G, Santeusanio F, Porcellati C, Brunetti P (2000) Relation between serum uric acid and risk of cardiovascular disease in essential hypertension. The PIUMA study. Hypertension 36:1072-1078

8. Davis TM, Zimmet P, Davis WA, Bruce DG, Fida S, Mackay IR (2000) Autoantibodies to glutamic acid decarboxylase in diabetic patients from a multi-ethnic Australian community: the Fremantle Diabetes Study. Diabet Med 17:667-674
9. Norman PE, Davis WA, Bruce DG, Davis TM (2006) Peripheral arterial disease and risk of cardiac death in type 2 diabetes: the Fremantle Diabetes Study. Diabetes Care 29:575-580

10. Holman CD, Bass AJ, Rouse IL, Hobbs MS (1999) Populationbased linkage of health records in Western Australia: development of a health services research linked database. Aust N Z J Public Health 23:453-459

11. Levey AS, Bosch JP, Lewis JB, Greene T, Rogers N, Roth D (1999) A more accurate method to estimate glomerular filtration rate from serum creatinine: a new prediction equation. Modification of Diet in Renal Disease Study Group. Ann Intern Med 130:461-470

12. UK Prospective Diabetes Study (UKPDS) Group (1998) Intensive blood-glucose control with sulphonylureas or insulin compared with conventional treatment and risk of complications in patients with type 2 diabetes (UKPDS 33). Lancet 352:837-853

13. Yusuf S, Hawken S, Ounpuu S et al (2004) Effect of potentially modifiable risk factors associated with myocardial infarction in 52 countries (the INTERHEART study): case-control study. Lancet 364:937-952

14. Clifford RM, Davis WA, Cull CA, Bruce DG, Batty KT, Davis TM (2004) Greater use of insulin by southern European compared with Anglo-Celt patients with type 2 diabetes: the Fremantle Diabetes Study. Eur J Endocrinol 151:579-586

15. Bruce DG, Davis WA, Davis TM (2000) Glycemic control in older subjects with type 2 diabetes mellitus in the Fremantle Diabetes Study. J Am Geriatr Soc 48:1449-1453

16. Culleton BF, Larson MG, Kannel WB, Levy D (1999) Serum uric acid and risk for cardiovascular disease and death: the Framingham Heart Study. Ann Intern Med 131:7-13

17. Fang J, Alderman MH (2000) Serum uric acid and cardiovascular mortality the NHANES I epidemiologic follow-up study, 19711992. National Health and Nutrition Examination Survey. JAMA 283:2404-2410

18. Tsouli SG, Liberopoulos EN, Mikhailidis DP, Athyros VG, Elisaf MS (2006) Elevated serum uric acid levels in metabolic syndrome: an active component or an innocent bystander? Metabolism 55:1293-1301

19. George J, Struthers AD (2008) The role of urate and xanthine oxidase inhibitors in cardiovascular disease. Cardiovasc Ther 26:59-64

20. Norman PE, Semmens JB, Laurvick CL, Lawrence-Brown M (2003) Long-term relative survival in elderly patients after carotid endarterectomy: a population-based study. Stroke 34:e95-e98

21. Bradshaw PJ, Jamrozik K, Jelfs P, Le M (2000) Mobile Australians: a moving target for epidemiologists. Med J Aust 172:566

22. Proctor PH (2008) Uric acid and neuroprotection. Stroke 39:e126

23. Lippi G, Montagnana M, Franchini M, Favaloro EJ, Targher G (2008) The paradoxical relationship between serum uric acid and cardiovascular disease. Clin Chim Acta 392:1-7

24. Kuzuya M, Ando F, Iguchi A, Shimokata H (2002) Effect of aging on serum uric acid levels: longitudinal changes in a large Japanese population group. J Gerontol A Biol Sci Med Sci 57:M660-M664

25. Jee SH, Lee SY, Kim MT (2004) Serum uric acid and risk of death from cancer, cardiovascular disease or all causes in men. Eur J Cardiovasc Prev Rehabil 11:185-191

26. Krishnan E, Svendsen K, Neaton JD, Grandits G, Kuller LH (2008) Long-term cardiovascular mortality among middle-aged men with gout. Arch Intern Med 168:1104-1110

27. Sakata K, Hashimoto T, Ueshima H, Okayama A (2001) Absence of an association between serum uric acid and mortality from cardiovascular disease: NIPPON DATA 80, 1980-1994. National Integrated Projects for Prospective Observation of Non-communicable Diseases and its Trend in the Aged. Eur J Epidemiol 17:461-468 